\title{
THE ORIGIN OF HIGH SPECIFIC FREQUENCY GLOBULAR CLUSTER SYSTEMS
}

\author{
M.G. LEE \\ Department of Astronomy, Seoul National University \\ Seoul 151-742, KOREA \\ AND \\ D. GEISLER \\ KPNO/NOAO \\ 950 N. Cherry Av., Tucson, AZ 85719 USA
}

There are known to be several giant elliptical galaxies with high globular cluster specific frequencies, which possess about three or more times the normal number of globular clusters for their luminosity. The origin of high specific frequency globular cluster systems is not yet known.

We have performed a definitive test of the idea of an intracluster origin for the high specific frequency by investigating the globular cluster system in NGC 4696. NGC 4696 is a giant elliptical, but not a cD galaxy, located at the dynamical center of the rich Centaurus cluster. The intracluster origin scenario predicts about 6 times more globular clusters than normal for its luminosity.

The luminosity function of the globular clusters shows a peak at $T_{1}=$ $24.5 \pm 0.1$. Comparing this value with that of the Galactic globular clusters, we estimate the distance to NGC 4696, obtaining $(m-M)_{0}=32.1 \pm 0.2$ for the reddening of $E(B-V)=0.12(d=26 \mathrm{Mpc})$. Incompleteness is not significant up to $T_{1} \sim 24.8 \mathrm{mag}$. Using the luminosity function of the globular clusters, we estimate the total number of globular clusters to be $N_{\mathrm{T}}=4100 \pm 200$. From this value and the absolute magnitude of NGC $4696\left(M_{\mathrm{V}}^{\mathrm{T}}=-22.1\right)$, we derive a value for the globular cluster specific frequency: $S_{\mathrm{N}}=6$. Then the excess number of globular clusters in NGC 4696 compared with $S_{\mathrm{N}}=4$ case is $\approx 1400$. This value is much lower than that expected by the intracluster origin theory, $\approx 20,000$. This result rules out the hypothesis of an intracluster origin for the high specific frequency globular cluster systems. 